Itinéraires Itinéraires

Littérature, textes, cultures

2020-3 | 2021

Le rap, une poésie de performances

\title{
La bûche et le transpalette : poétique d'Odezenne
}

Poetics of Odezenne

Denis Saint-Amand

\section{OpenEdition}

Journals

Édition électronique

URL : https://journals.openedition.org/itineraires/8788

DOI : $10.4000 /$ itineraires.8788

ISSN : 2427-920X

Éditeur

Pléiade

Référence électronique

Denis Saint-Amand, «La bûche et le transpalette : poétique d'Odezenne », Itinéraires [En ligne], 2020-3 I 2021, mis en ligne le 09 décembre 2021, consulté le 12 décembre 2021. URL : http://

journals.openedition.org/itineraires/8788; DOI : https://doi.org/10.4000/itineraires.8788

Ce document a été généré automatiquement le 12 décembre 2021.

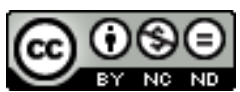

Itinéraires est mis à disposition selon les termes de la licence Creative Commons Attribution - Pas d'Utilisation Commerciale - Pas de Modification 4.0 International. 


\title{
La bûche et le transpalette : poétique d'Odezenne
}

\author{
Poetics of Odezenne
}

Denis Saint-Amand

\section{À distance du bad boy}

Dans son Histoire du rap en France, Karim Hammou met en lumière la façon dont les logiques oppositionnelles emblématiques des champs culturels contribuent à la constitution du milieu du rap français comme espace de luttes (labels indépendants vs majors, légitimité vs succès de masse, etc.), et trouvent dans les années 2000 à se prolonger par «une profusion de conflits dans lesquels les normes de la rue sont explicitement invoquées et dont les enjeux implicites ont souvent partie liée à des conflits économiques directs entre protagonistes, qu'il s'agisse de relations de production jugées préjudiciables ou de rivalités commerciales" (Hammou 2012: 242-243). Cette modélisation efficace permet de rendre compte d'une systématisation de la querelle comme principe dynamique inhérent à l'univers du rap français ${ }^{1}$ - en témoigne, parmi d'autres, le conflit entre Booba et Kaaris, concrétisé tant sur le plan discursif (par clashs interposés) que physique (lors de la bagarre à l'aéroport d'Orly, au mois d'août 2018) et bénéficiant d'une large exposition médiatique.

2 Si l'univers du rap français est lourdement chargé d'une dimension polémique pesant sur l'imaginaire qu'il entretient, nombreux sont aussi les artistes qui s'en affranchissent, investissant des thèmes, formes et procédés permettant de déplacer les enjeux et fonctions d'un univers musical, mais aussi de contribuer à sa démocratisation. Le comble de cet apaisement est sans doute incarné par le duo Bigflo \& Oli, qui, sur le titre «Gangsta » (2014), revendique son absence de street cred' en renversant un imaginaire belliqueux stéréotypé et en brisant la logique de l'ego trip pour affirmer une banalité fonctionnant paradoxalement comme vecteur de distinction ( J'suis pas un gangsta / Pas la peine de mentir, j'suis comme toi / Freestyle sous le préau ») : cette constitution d'un ethos humble permet aux deux frères de se tenir à l'écart d'une 
représentation du rap dont ils infèrent qu'elle constitue une norme (participant en cela à l'entretien des stéréotypes sur cet univers), d'être perçus comme fréquentables par le grand public et de favoriser la réception de leurs productions au sein du cadre familial. Sans aller jusqu'à un tel lissage ${ }^{2}$, plusieurs artistes issus de la scène rap française ont développé une production misant sur la dérision vis-à-vis de la mythologie du bad boy. C'était déjà le cas chez IAM, dont l'emblématique « Petit frère » (1997) dépeignait avec ironie l'empressement d'un aspirant caïd, chez Stomy Bugsy (la prosopopée « Mon papa à moi est un gangster ", 1996) et Doc Gyneco (“Classez-moi dans la variet” », 1996) en marge de leurs productions plus radicales avec le Ministère A.M.E.R., ou encore chez Disiz' La Peste, dont le titre à succès "J'pète les plombs " (2000) est une réécriture ludique du thriller Falling Down de Joel Schumacher (1993). La tendance se développe dans les années 2010, en entretenant ce qu'Anthony Pecqueux (2007 et 2008) présente comme une forme de nonchalance articulatoire et posturale. Cette stratégie énonciative est adoptée, entre autres, par Orelsan et Gringe, dont les productions (individuelles comme celles qu'ils signent ensemble sous le nom des Casseurs Flowters) relativisent leur position privilégiée en misant sur la marginalité, l'ennui et l'échec, qu'ils assument au second degré : omniprésente dans leurs textes ${ }^{3}$, cette posture est en outre prolongée dans le film Comment c'est loin (réalisé par Orelsan et Christophe Offenstein, 2015) et la série Bloqués, diffusée de septembre 2015 à août 2016 sur Canal +, dans lesquels les deux rappeurs jouent leur propre rôle et donnent à voir les misères de leur vie quotidienne. Une démarche voisine est adoptée par Vald, qui, dans le titre «Bonjour » (2015), ironise sur la violence associée au milieu du rap en relatant le destin funeste d'un passant discourtois ( Il a pas dit bonjour / Du coup, il s'est fait niquer sa mère $»)$, tout en raillant les déformations des récits de ces hauts faits pour mieux conclure sur une parodie de message préventif insistant sur l'importance de la politesse. Elle s'observe enfin chez Lorenzo, qui, pour qualifier son travail, va jusqu'à mobiliser l'étiquette générique au second degré de "troll rap » : l'artiste, de son vrai nom Jérémie Serrandour, a suivi une terminale littéraire avec option théâtre et cinéma au lycée Bréquigny de Rennes, où il a obtenu son baccalauréat en 2013. Le personnage qu'il campe sous le pseudonyme de Lorenzo est reconnaissable à son bob «Game Boy Color ", à ses lunettes de soleil et à sa voix nasillarde, vectrice d'un idiolecte misant sur la forgerie ("mamène », calque de l'anglais «my man ») et la réhabilitation d'adjectifs désuets (« cocasse »). Il se présente comme «l'empereur du sale », façon de baliser un projet fictionnel à la fois flou et singulier («faire du sale ») qui se concrétise à travers l'accumulation de conquêtes amoureuses et la consommation de psychotropes. Le titre qui a révélé l'artiste, "Freestyle du sale ( (2016), jouait d'une poétique chamarrée rappelant les soliloques cyniques d'un Kaaris (entre hyperboles - «J'suis le grand frère de Pascal le grand frère »; "J'ai 86 enfants et 132 femmes »; « À l'école c'était moi qui venais donner les ordres »-, évocation plus comique que menaçante de la délinquance - «J't'envoie ta commande par pigeon voyageur, t'inquiète, ils sont sous coke, ça arrive dans l'quart d'heure » - et invectives périphrastiques - «J'ai niqué la sœur de la mère de ta cousine »). Depuis lors, Lorenzo mise davantage sur une dérision propre à l'esprit $\mathrm{du}$ trolling des réseaux sociaux, dont témoignent les vidéos humoristiques qu'il poste sur sa chaîne YouTube ("Lorenzo à l'usine de cds", "La maison hantée de Mike Douglas », «La météo du sale ») et des titres comme " Fume à fond », "Carton rouge » ou « Nique la BAC».

3 Tenant également toute possibilité d'héroïsation à distance, le groupe bordelais Odezenne, formé par Alix Caillet, Jacques Cormary et Mattia Lucchini (tous trois nés en 
1981), a lui aussi réussi à se distinguer en proposant, depuis le milieu des années 2000, une alternative à la production dominante en matière de rap français. Lorgnant volontiers vers le rock, comme en témoignent à la fois l'historique du projet ${ }^{4}$, certaines des influences qu'il revendique ${ }^{5}$ et les arrangements qu'il développe en studio et sur scène, Odezenne $a$, en un peu plus d'une dizaine d'années d'existence, livré quatre albums (sans. chantilly, 2008; OVNI - pour "Orchestre Virtuose National Incompétent »-, 2011; Dolziger Strasse 2, 2015; Au Baccara, 2018 - avant 1200 mètres, dont la sortie est prévue le 7 janvier 2022) et deux EP (Rien, 2014 et Pouchkine, 2019). Le groupe doit notamment sa position singulière dans le champ contemporain à une stratégie de production et de diffusion délibérément oblique : ses disques sont tous plus ou moins autoproduits et signés sur Universeul, label au nom parodique fondé par le trio, qui se passe fort bien de programmation radio et qui rend dispensables les stratégies réticulaires exploitées par les grandes maisons à coups de featurings et autres collaborations entre acteurs du champ ${ }^{6}$. Les rapports d'Odezenne avec les sociétés de booking sont eux aussi particuliers : s'il s'entoure ponctuellement de labels pour assurer leur circulation à l'étranger (comme Nada Booking pour la Belgique), le groupe a souvent préféré passer outre les promoteurs français, développant sur les réseaux sociaux un système participatif («Odezenne à la demande ») qui a permis l'organisation de plusieurs tournées depuis 2013. Cet investissement de circuits parallèles favorise la connexion avec un public dont l'opinion est sollicitée et prise en considération, et renforce en toute logique l'adhésion de ce dernier, qui se trouve engagé dans un rapport d'échange que les concerts portent à leur comble ${ }^{7}$.

Ce qui m'intéressera dans les pages qui suivent est la façon dont, en sus de cette singularité réticulaire et artisanale, Odezenne s'est forgé une identité propre à la faveur de ses textes. Il s'agira d'essayer de saisir la poétique du groupe, en interrogeant les tendances formelles qu'actualisent son écriture et la façon dont celles-ci se mettent au service d'un imaginaire et d'un système de valeurs défendus par le trio.

\section{Jeux rhétoriques et style potache}

5 Dès l'album sans. chantilly, Odezenne (et, en premier lieu, Alix Caillet, préposé à la rédaction) propose une écriture soucieuse de se distinguer par un emploi nourri de figures de style et, en particulier, de celles qui produisent un écart ou une saillance sur le plan sonore - soit celles que le Groupe $\mu$ désigne comme métaplasmes ${ }^{8}$. La récurrence de ces procédés n'empêche pas le trio de se fonder sur des thématiques universelles (le poids des souvenirs dans " Danse des morts ", le déclin de l'époque mesuré à l'aune de la population des villes dans « En bas de ma fenêtre » et « Mr 2007 », ou le triomphe du conformisme dans «Dis-moi » et «Existe petit bout de rien»), ni de construire des micro-fictions urbaines (comme "Destins croisés", une prosopopée énoncée par un personnage découvrant que la femme mariée avec qui il entretient une liaison le trompe avec un autre). L'omniprésence des procédés métaplasmiques semble toutefois leur conférer un rôle spécifique, quasi performatif, comme manière d'exhibition garantissant le droit d'entrée dans le champ: l'accumulation de paronomases, allitérations et autres jeux d'échos par chiasmes, rimes et assonances tient de l'épreuve de force, avalise les aptitudes techniques du trio émergent et favorise la représentation d'un collectif doué sur le plan rhétorique. En témoignent, parmi d'autres, des titres comme «Tic-tac» («Lac de larme antique, / De bric-à-brac, /J'braque ma case en 
briques»), «Feuille blanche» («Principe des vases communicants / Commun mais quand ? / L'commun m'niquant, / Comment communiquer/Si l'unique point manquant commun manquait?») ou « Destins croisés ( «Blesse mon estime, mais estce de trop? / Épine épaisse: Damoclès / Épée ou pas: une peste, ma nana/Que j'bouffais comme une split banana... »).

6 Si cette dimension métaplasmique se réduit au fil des albums, elle ne disparait jamais totalement et s'articule à d'autres effets et enjeux. Sur OVNI, le morceau « Meredith" est de cette façon rythmé par une poétique de la paronomase qui soutient le portrait d'une femme en perte de repères ("Dans les clubs elle déboule, / de la bave en découle, / ses pelles roulent, / elle est saoule, / elle roucoule sous la boule... / Fait du zèle, zèle, zèle,/ brûle ses ailes, ailes, ailes ») que la domination masculine et le sentiment de solitude poussent au suicide («Un pied sur une chaise, / de l'air sur le visage, / un corps en terre glaise, / elle regarde des images. / Meredith, -dith, -dith, / tu nous quittes, quittes, quittes? / Elle plonge du cinquième un sourire aux lèvres »), tandis que le bonus explicite « Tu pu du cu » est l'occasion d'une invective au second degré; adoptant le ton des joutes oratoires inhérentes aux scènes de battles, l'énonciateur se présente en esthète face à un adversaire imaginaire qu'il attaque et que la verve du freestyle est censée désarmer ("T'es stupéfiant/Je te roule/Je te fume / T'es stupéfait / Je suis la lune / Et toi l'bitume / T'es cétacé / T'es assassin / T'es fracassé / T'es ramassé / J'suis fricassé / Toi pique-assiette / J'suis Picasso / T'es Robinson »). Cette logique énonciative accordant une large place au jeu participe à la mise en place de ce que je propose d'appeler un style potache, c'est-à-dire un ensemble de prises discursives et comportementales volontiers ludiques et convergentes, fondées sur l'intérêt au désintérêt, la défiance du caractère normatif des institutions, l'opposition aux systèmes dominants et l'irrévérence désinvolte9.

7 Un titre comme «Saxophone » est construit comme un exercice de style sur le modèle des associations "Marabout-bout de ficelle", ces concaténations paroxystiques si chères à Franquin et Delporte ("Saxophone / phonographe / graff" de fou / foule de gens »), tandis que «Bûche », dévoilé pour les fêtes de la fin d'année 2013 et s'ouvrant sur un sample de « Happy Holyday » de Bing Crosby (1942), se construit, par assonances et calembours, sur le modèle du portrait chinois («Si j'étais un super héros / J’serais mélomane / Si j'étais une pièce / J'serais un hammam »; "Si j'étais un bouquin / Je serais Les Fleurs du mal / Si t'étais un bouquet / Tu serais les fleurs du mâle ») avant que le refrain ne se présente comme une opposition frontale aux forces de l'ordre sur la base d'une comparaison a priori absurde transformant, par dérision et en exploitant la polysémie du mot bûche, un cliché de Noël en projectile de manifestant : «Et si j'étais une bûche / Je ferais du saut à ski sur la gueule d'un CRS ». Parce qu'il produit un effet de rupture au cœur d'un pseudo-chant de Noël en y introduisant une image violente et que cette rupture est elle-même provoquée par un jeu calembouresque, le refrain de « Bûche » oscille entre la potacherie et l'activisme; il peut s'envisager comme un pivot dans la trajectoire d'Odezenne, dont les textes ultérieurs déclineront davantage les motifs de la contestation et de l'engagement social.

8 La maîtrise d'une rhétorique métaplasmique a permis au groupe d'émerger et de se conférer une image de techniciens habiles roués aux virtuosités phoniques; si, en évoluant, le groupe a exploité des procédés différents, il reste attaché à cette forme d'écriture privilégiant l'homophonie et la paronomase, qu'il continue à exploiter sur Dolziger Strasse 2 avec des titres comme «Vilaine» («Mon alcool colle au sol / Mes 
semelles, s'mêlent au ciel / La blonde onde du corps, / ma tête tète la bouteille ») et "Corps à prendre » («Rien de plus, tout me va, / saut de puce où je vais / Rien ne m'use ici bas/ tout compte fait »), mais aussi sur Au Baccara avec « Pastel» («Pastel / Rien à fouler / Besoin d'elle / Rien à fourrer / Pas de chrysanthème / Rien à rouler / J'reste seul / Rien à branler »), le titre éponyme («Jeûner, jeûner, jeûner, jeûner/Je n'ai jamais faim / Tune, tune, tune, tune, tune / Tu ne tueras point / L'ennui, l'ennui, l'ennui, l'ennui, / La nuit ce dégât ») et, surtout, " Nucléaire », dont les deux couplets ne se distinguent que par un jeu de permutation entre les derniers mots de chaque vers (de cette façon, les quatre premiers vers « Comme tu m'éclaires au nucléaire j'ai le ciel bleu / Tu me ramènes quand je déraille un jour sur deux / Le temps qui passe, sans ton visage, c'est pas heureux / J'aime pas compter, j'aime pas savoir qui gère le jeu » deviennent, dans le deuxième couplet, «Comme tu m'éclaires au nucléaire j'ai le ciel vieux / Tu me ramènes quand je déraille les jours heureux / Le temps qui passe, sans ton visage, c'est pas du jeu /J'aime pas compter, j'aime pas savoir qui gère le feu »).

\section{Hédonisme et complicité}

Au-delà des démonstrations rhétoriques, les morceaux d'Odezenne se caractérisent sur le plan du contenu par l'affirmation d'une axiologie fondée, d'une part, sur une conduite mêlant paresse et hédonisme, et, d'autre part, sur l'importance des relations privilégiées au sein même de cette culture du plaisir. L'hédonisme d'Odezenne se construit en opposition à une représentation de la norme, des obligations sociales et de la routine inhérente au monde du travail, qui apparait comme un épouvantail pour de nombreux artistes liés à l'univers du rap français - c'est notamment le cas pour Orelsan et Gringe ( «Abattu par la fatigue d'avoir rien branlé / Le projet c'était d'rien foutre, et j'ai aucun plan B », sur « Regarde comme il fait beau », parmi de multiples exemples), pour 1995 ( «J'ai la flemme de travailler, de batailler et puis d'me raser le bouc», sur «La flemme ») ou pour le Klub des Loosers («À mon taf on m'appelle l'indien : toujours bourré dans la réserve! / Tu seras gentil, fous-moi la paix. Pour que mon calumet serve / J'envoie des signaux de fumée super faciles à décrypter / Vu qu'ils veulent tous dire la même chose : / Évitez de me parler ", sur " Indien »).

La thématique de la distinction, revendiquée comme manière d'échapper à l'orthodoxie, est exploitée dès le premier album, entre autres sur le morceau «Dismoi ", où un portrait du "modèle dominant qui te domine", figuré par le citoyen conforme et banal, est esquissé comme repoussoir ( $\mathrm{J}$ 'caresse, des moments d'vérité / mais tard le soir j'suis dérouté / laisse tomber la sincérité / s'insérer dans l'rang serré / à commencer par l'jean serré / les ch'veux gominé, c'est / le modèle dominant qui te domine »). Pour résister à cette norme accablante et s'en émanciper, le trio, sans miser sur la possibilité d'un bonheur de tous les instants («Aimer tous les moments / C'est s'mentir dans le sens / C'est s'mentir dans le froid / C'est noyer son essence » sur "On nait, on vit, on meurt»), exploite le recours au plaisir sexuel (par exemple, sur le single " Je veux te baiser ", devenu l'un des hymnes du groupe), tout en thématisant les apories et déconvenues sur lesquelles il peut déboucher (sur des titres comme "Destins croisés ", «Plus beau cul du monde" ou « Nasty»), et mobilise les paradis artificiels comme échappatoires parégoriques ( J'ai collé deux feuilles en L / pour oublier les fêlés / qui font tout péter pêle-mêle ", sur «En L»), non sans évoquer, là encore, les problèmes qu'ils peuvent générer ("Vodka! Dans un gros 
tonneau / Attaquer mon foie / Au chalumeau / Souder les sanglots», sur «Vodka»; «Bébé m'a dit maudit! / Tu-tu n'aurais pas dû / Ramener ta gueule ici / Dedans y'a du beau monde / Et toi t'as les yeux troubles », sur « Bébé »).

11 Sans renier ces thèmes qui permettent d'offrir des pauses respiratoires dans un monde perçu comme asphyxiant, le groupe semble avoir, depuis au moins Dolziger Strasse 2, trouvé une autre manière de résister au marasme dans l'éloge du lien fort, de l'affinité élective : là où l'énonciation des premiers textes était principalement dévolue à un "Je » et où la deuxième personne du singulier était surtout apostrophée au détour d'une invective ou récrimination (sur « $\mathrm{Tu} \mathrm{pu} \mathrm{du} \mathrm{cu}$ » ou «Dieu était grand», par exemple), les productions accueillies sur les troisième et quatrième albums accordent au «nous» la possibilité de se déployer et semblent lui conférer une mission thérapeutique. Le cas est particulièrement manifeste sur le titre "Souffle le vent », qui peut s'appréhender comme une invitation au voyage, cherchant dans la complicité avec l'être aimé la possibilité de résister aux affres de la médiocrité quotidienne :

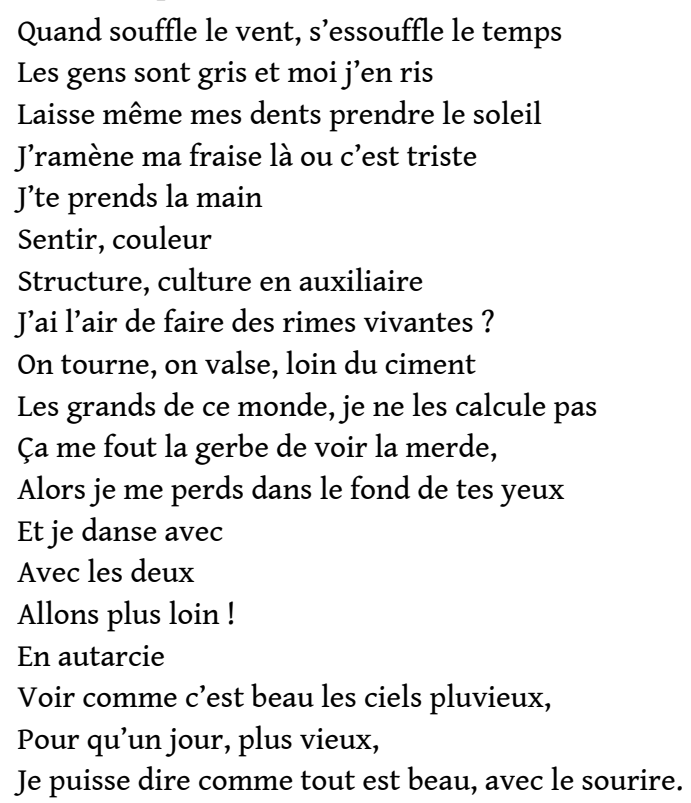

12 Sans se départir d'une tendance au calembour («pluvieux»/ "plus vieux»; l'expression « en autarcie » qui, suivant l'injonction « Allons plus loin!», paraitt perdre son sens premier pour devenir une improbable destination exotique) et à la réflexivité ironique («J'ai l'air de faire des rimes vivantes?»), le groupe semble ici redéfinir sa vision du monde en délaissant l'expérience de la déréliction et le constat désabusé d'un affaissement généralisé pour une reprise en main énergique fondée sur la croyance en la fuite vers des lendemains meilleurs. Le départ implique une rupture avec une grisaille urbaine vis-à-vis de laquelle l'énonciateur se trouve en porte-à-faux (comme le signale l'antithèse « les gens sont gris et moi j'en ris ») et implique un déplacement vers un ailleurs fantasmatique propre à un imaginaire quasi romantique, permettant la liberté des corps et la reconquête de la nature ( On tourne, on valse, loin du ciment »). La concrétisation de cet idéal peut se lire dans «Matin ", titre figurant sur l'EP Pouchkine (2019) mais sorti trois ans auparavant, à l'automne 2016, et escorté alors par un clip de Jérôme Clément-Wiltz. Ce dernier donne à voir un couple de jeunes marins, Hannah et Mathurin, vivant dans une petite cabane située près des Roches-Blanches, à Douarnenez, en Bretagne. La relation amoureuse figurée dans la vidéo oriente la réception d'un texte qui, considéré isolément, peut sembler consacré à l'amitié : « Dans 
nos yeux / Dans nos deux / Y a la vie, mon ami, faute de mieux / On verra bien / Jusqu'à quand / On dirait qu'on défie les heureux ». Ici aussi, le couple est situé en marge d'un univers forcément décevant qu'il s'échine à déserter, et c'est dans la complicité amoureuse que résident les seules raisons de croire encore à l'avenir.

Pareil sentiment s'observe dans « Nucléaire » où, nous l'avons vu, la fusion amoureuse est supportée, d'une strophe à l'autre, par un système de reprise formelle avec interversion des termes à la rime rappelant par mimétisme l'entrelacement amoureux, ou dans "Tony", onzième et dernier titre d'Au Baccara, qui s'énonce comme une promesse : « Collée à moi tu ne briseras jamais / J'aurai toujours pour toi / Des mots, du beau, / De jolis mois de mai / Collée à moi tu ne tomberas jamais / J'aurai toujours pour toi, la paix, / De quoi terrasser la misère ». S'il s'engage à protéger celle qu'il aime et à éclaircir son quotidien, l'énonciateur n'en infère pas moins que le monde dans lequel ils évoluent est morne et affligeant : la position qu'il se propose d'occuper est celle d'un rempart ou d'une béquille, aidant à supporter les assauts et obstacles de l'extérieur l'optimisme du trio ne portant pas sur l'état du monde - présenté comme insupportable depuis sans.chantilly -, mais sur la capacité de triompher de celui-ci dans la communion amoureuse. Dans "Tony» toujours, les promesses faites à l'aimée impliquent «de jolis mois de mai » : si la formule peut s'envisager au pied de la lettre, comme l'annonce de printemps radieux à venir, elle ne peut manquer de renvoyer à Chris Marker ${ }^{10}$ et à l'insurrection de 1968. Que ces échos soient délibérés ou non, ils permettent d'assurer la transition vers une troisième dimension de la poétique d'Odezenne, qui tient à l'engagement du propos dans le débat sociopolitique contemporain.

\section{Du rire à l'engagement}

L'EP Rien, sorti en 2014, a permis d'amorcer un tournant singulier dans la trajectoire d'Odezenne : si, dans son rapport au monde social, le trio s'en était jusqu'alors tenu à des considérations résolument générales sur la position de ses membres dans leur époque - considérations empreintes d'un sentiment de malaise, sinon de déréliction, et dialoguant avec les productions d'autres artistes associés à la même veine, comme on a pu le voir -, cette plaquette change partiellement la donne en octroyant une place, aux côtés de titres prolongeant la conduite hédoniste ( Je veux te baiser ») et l'esthétique du ratage («Rien », " Dieu était grand »), à deux titres, « Novembre » et "Chimpanzé », dont le propos est plus directement arrimé à des enjeux sociopolitiques.

« Novembre » sera accompagné, en juin 2016, par une vidéo de Jérôme Clément-Wilz, prenant pour objet les manifestations des membres de "Nuit Debout» devant l'Assemblée nationale contre la «loi travail». Le morceau s'ouvre sur une double condamnation fustigeant deux figures érigées en responsables du marasme contemporain ( C'est avec les ambitions qu'on attrape le financier/Avec les munitions que dérape le policier»), avant de prendre des allures de chant révolutionnaire annonçant une insurrection à venir («La détresse est visible elle se couche dans nos lits /[...] Ils ont repris la vie de n'importe quelle façon/De l'accumulation, naît la révolution»). Le morceau se distingue des productions précédentes d'Odezenne par son engagement frontal et sa détermination; guère de jeu de mots potache ou d'hymne hédoniste ici, mais la prise en considération d'une crise dont les signes se multiplient et éreintent. « Notre armée triomphante est rentrée dans 
Paris ", signale l'énonciateur, et le constat peut rétrospectivement sembler prophétique, tant il paraît annoncer l'avènement des Gilets jaunes ${ }^{11}$ - ce n'est d'ailleurs pas un hasard si certains membres du mouvement, dans les écritures sauvages qui ont accompagné leur contestation, sur les murs des villes ou sur leurs propres gilets, ont plusieurs fois emprunté à "Novembre» le vers "Une étincelle brille entre deux explosions », engageant de cette manière un dialogue avec le groupe.

Figure 1. "Une étincelle brille entre deux explosions », janvier 2019.

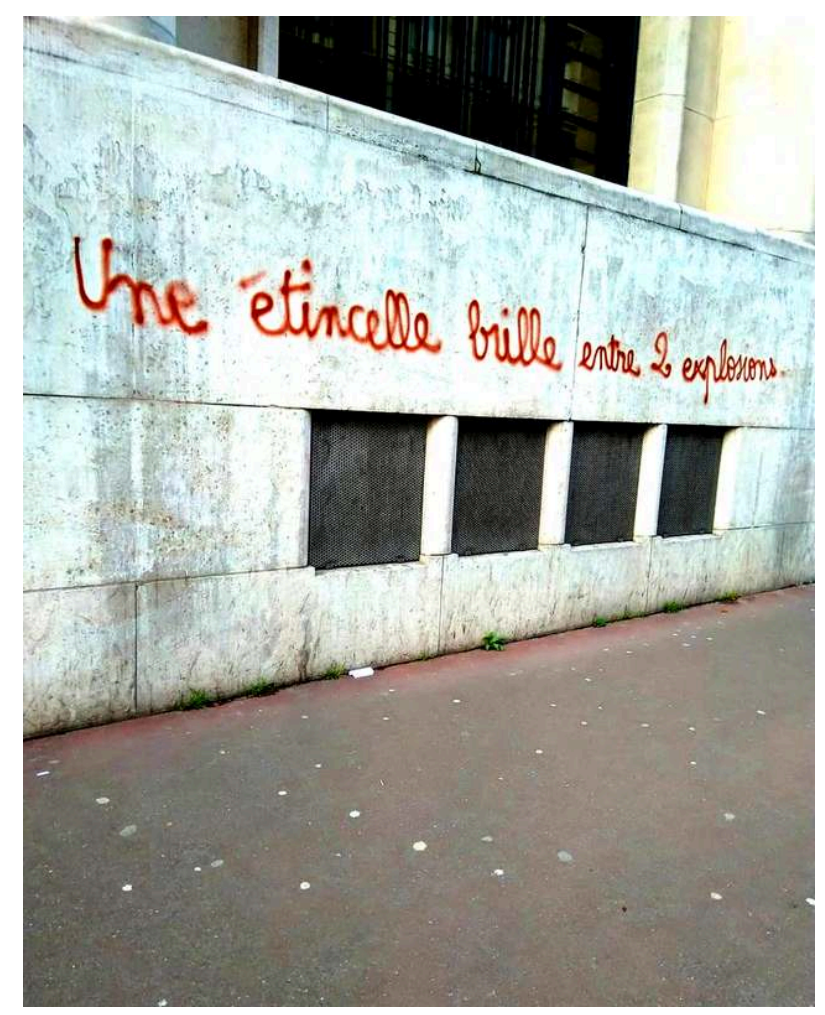

Source : Tumblr, La rue ou rien, https://larueourien.tumblr.com/.

L'autre titre politique figurant sur Rien, "Chimpanzé », peut s'envisager comme une prosopopée énoncée par un individu en train de perdre pied, oscillant entre fébrilité et exaspération ( "J'ai le crâne circulaire brumeux comme l'horizon / Qui sniffe de la poudre à canon ", dit-il dès l'incipit), et dont on saisit progressivement la précarité. Agressé par des éléments naturels qui deviennent hostiles parce qu'il ne peut s'en protéger (« La pluie ronge les réverbères comme le coton / [...] Désespoir à l'eau de vie qui brûle comme le Destop »; «Et le soleil me mord, j'dis qu'il a tort / Et lui tords le cou avec mes deux pouces »), le sujet multiplie les allusions à des situations d'exclusion. La sienne peut sembler celle d'un réfractaire refusant de s'inféoder aux exigences d'une société capitaliste (comme le soutient une antithèse mâtinée d'un calembour douloureux: "La tête vissée sur l'espérance, je ne suis pas niais/Je suis nié, car j'crache à la gueule des mecs sur les billets »), mais l'énonciateur sait qu'il n'est pas le seul laissé pour compte : il s'en prend à l'Église au détour d'une synecdoque, soulignant l'hypocrisie d'une institution préférant amasser les richesses plutôt qu'œuvrer à la mission de justice sociale qui lui est dévolue («Une question à résultats pour être positif / Quitte à pas être égal entre le Vatican et le Sénégal»). Là où «Bûche " se métamorphosait de titre comique en morceau activiste par la force d'un refrain 
calembouresque et virulent, «Chimpanzé » se charge d'une isotopie de la violence pour dénoncer autant celle provoquée par la distribution inégale du capital que celle exercée par les appareils répressifs d'État, à travers des motifs saisissants («Une patate sans gant, une trique de keuf sanglante "; "Une grenade juteuse dans les jambes d'un enfant / Mort de naissance devant l'assistance publique / Sa mère en fait-divers dans un papier d'encre »). Dans le même ordre d'idées, la référence à "Stalingrad qui crame sous trente-quatre degrés centigrades " ne renvoie pas tant à l'ancien nom de la ville russe de Volgograd qu'à la station de métro parisienne, à la limite des $10^{\mathrm{e}}$ et $19^{\mathrm{e}}$ arrondissements, aux alentours de laquelle vivotent de nombreux toxicomanes "Stalingrad qui crame" n'étant pas sans rappeler, par paronomase, le "crack", "came» que l'on «crame» (la station est parfois surnommée "Stalincrade » ou «Stalincrack » par les riverains). Surtout, c'est là que se sont improvisés, depuis 2015, des camps de réfugiés contraints de s'installer dans des conditions déplorables et souvent évacués manu militari par la police. La vidéo escortant le morceau, sortie en septembre 2016, permet de recharger le texte (composé deux ans plus tôt) de cette thématique migratoire : réalisée par Arthur Muller, qui a contacté le groupe après avoir découvert le clip de "Novembre ", elle consiste en un micro-documentaire suivant des familles fuyant leur pays, depuis l'île de Lesbos jusqu'à la frontière entre la Serbie et la Hongrie, où la politique d'« accueil » de Viktor Orbán s'est distinguée par sa violence et son ignominie.

Les textes d'Odezenne semblent de plus en plus fonctionner comme des prises de position et dépasser la revendication d'une marginalité pour dénoncer une série d'inégalités jugées insupportables. Sur Au Baccara, le groupe livre notamment «BNP », dont le seul titre renvoie à la banque française (la plus importante de la zone euro) dont la réputation est entachée par plusieurs polémiques liées, entre autres, à sa politique de licenciement ${ }^{12}$ et, comme l'a révélé l'affaire des "Panama papers", à son recours à des sociétés-écrans situées dans des paradis fiscaux ${ }^{13}$. Le morceau s'ouvre sur un soliloque prononcé par Nabounou, réfugiée béninoise ( J’ai rencontré des gens honnêtes, des malhonnêtes. Certains viennent causer avec toi, parler, te demander si ça va. Ils viennent montrer les dents, Jouer avec nos sentiments. Mais c'est la fausse bonne humeur. Ils sont mauvais dans leur cœur»), tandis qu'en arrière-fond le beat s'intensifie et que la voix d'Alix Caillet se superpose à celle de la jeune femme pour clamer «rends la monnaie/rends les billets!». Ce double slogan fait écho à l'injonction "Rends l'argent!", devenue virale au moment de l'affaire Fillon ${ }^{14}$. Le morceau se fonde sur une dynamique antithétique, mettant en parallèle l'expression d'une réfugiée désemparée et le cynisme d'institutions embarquées dans la course au profit économique, se souciant peu de la situation défavorable des individus alors que ceux-ci sont les premiers contraints aux restrictions lors de périodes de crises financières, comme ce fut le cas en 2008.

Enfin, le dernier exemple permettant de mesurer le tournant politique du groupe est le titre liminaire de l'EP Pouchkine, «Bleu fuchsia ». Sorti en avril 2019, alors que le groupe était en tournée, le morceau est chanté par Jacques Cormary et porte sur son expérience en tant que manutentionnaire au marché de Rungis : le texte, l'un des plus narratifs du groupe, esquisse un portrait du travailleur aliéné, écrasé par les horaires contraignants ( Je me souviens du bus de nuit / Direction le marché de Rungis / Sur le carreau je finis ma nuit / Au son du peul des mamas Mali»), éreinté par la pénibilité d'une tâche physique («Portant de lourds colis / Des cernes pleines sous les yeux»; 
« Décharger les cametards / Les muscles exultent »), rompu par le mépris et la violence exercés par le client et le patron (« Le client est pas rigolo / Il a des blagues salaces / Il renifle son nez d'alcoolo / Et il crache dans les salades »; "Neuf heures et quart / Le boss tout frais arrive et scrute/Il s'en fait pour son argent/Il fait vivre des familles / C'est ce qu'il me dit/Quand je fais tomber une paire de mangues/Ses pompes disent le contraire / Elles brillent au fond du container / Elles disent que j'ai mauvais goût / D'avoir des pulls, avec des trous»), las du racisme ambiant («Le vendeur me casse les glandes/Il aime pas bien la couleur/Du préparateur de commande ») et méfiant d'une accoutumance au milieu qui semble conduire tout droit à l'assommoir ( $\mathrm{Y}$ a plein de soûlots / Assommés au comptoir/Ils content les histoires / Ils comptent les goulots »). Rythmé par un refrain synthétisant la routine et la mélancolie qu'elle provoque en une manière de chronotope oppressant (« Le ciel est triste/Je trie des pommes»), le morceau s'ouvre toutefois par un autoportrait synecdochique et louangeur focalisé sur les mains du sujet: "Mes mains sont belles / Elles ont la forme du travail / Les ampoules de la tenaille / Pour les plombs que j'ai brisés ». Rappelant partiellement l'éloge des femmes de la Commune tissé par Rimbaud dans «Les mains de Jeanne-Marie » ("Ce sont des ployeuses d'échines, / Des mains qui ne font jamais mal, / Plus fatales que des machines, / Plus fortes que tout un cheval !»), cet incipit mélioratif peut s'entendre à la fois comme un encouragement à maintenir le cap et comme un geste honorifique, une manière d'hommage à l'ensemble des mains usées par la besogne et les sacrifices.

«"Bleu fuchsia", c'est notre côté gilets jaunes! » affirmaient les membres du groupe au Focus Vif à la sortie de l'EP ${ }^{15}$. Si le texte parvient à articuler éloge de l'abnégation des mains-d'œuvre et dénonciation des conditions de travail, il s'autorise un clin d'œil aux mouvements sociaux au détour d'un quatrain a priori anodin, mais chargé d'une double allusion complice: "Transpalette/Grand ballet/Je reste fier/De ma race ferroviaire ». Cette "race ferroviaire " à laquelle le sujet dit appartenir et dont il revendique la fierté, c'est évidemment celle des cheminots, dont on connaît l'importance dans les mouvements contre le « plan Juppé » en 1995 ; les mêmes qui, au printemps 2018, se sont mobilisés contre la réforme de la SNCF souhaitée par le gouvernement et qui, à l'heure où j'écris ce texte, s'inquiètent de la baisse des conditions de sécurité impliquée par le choix de faire circuler certains trains en « équipement agent seul » (EAS), c'est-à-dire sans autre agent à bord que le conducteur. Quant au distique "Transpalette / Grand ballet », s'il fait référence au chariot servant au déplacement de palettes de manutention - élément participant du décor du marché de Rungis -, il est surtout l'occasion de renvoyer, par une sorte de pied de nez, à l'attaque du ministère occupé par le secrétaire d'État et porte-parole du gouvernement, Benjamin Griveaux, par des Gilets jaunes équipés d'un engin de chantier, le 5 janvier 2019. Dès le lendemain, les membres et sympathisants de la mobilisation revendiquent cet assaut en nimbant sa violence d'une ironie ayant pour effet de la charger d'une dimension euphorique et de la distinguer de celle, froide, exercée par les forces de l'ordre. Sur les murs de la capitale, on voit surgir des graffitis du genre : "Encastrer la préf au transpalette», «Demain s'ouvre au Fenwick!", "Génération Fenwick!", «Rendez-nous le tractopelle!", "Macron démission, Transpalette à Matignon » et «Nous sommes transpalette», reprise ironique de la structure du slogan "Je suis Charlie", popularisé après l'attentat contre le journal satirique Charlie Hebdo du 7 janvier $2015^{16}$. Associant l'outil à un "grand ballet ", c'est-à-dire à un motif spectaculaire sinon festif, Odezenne s'inscrit dans le sillage des Gilets jaunes au détour 
d'une blague d'initiés permettant, à sa façon, d'affermir les liens d'une communauté insurgée.

Figure 2. « Nous sommes transpalette », janvier 2019.

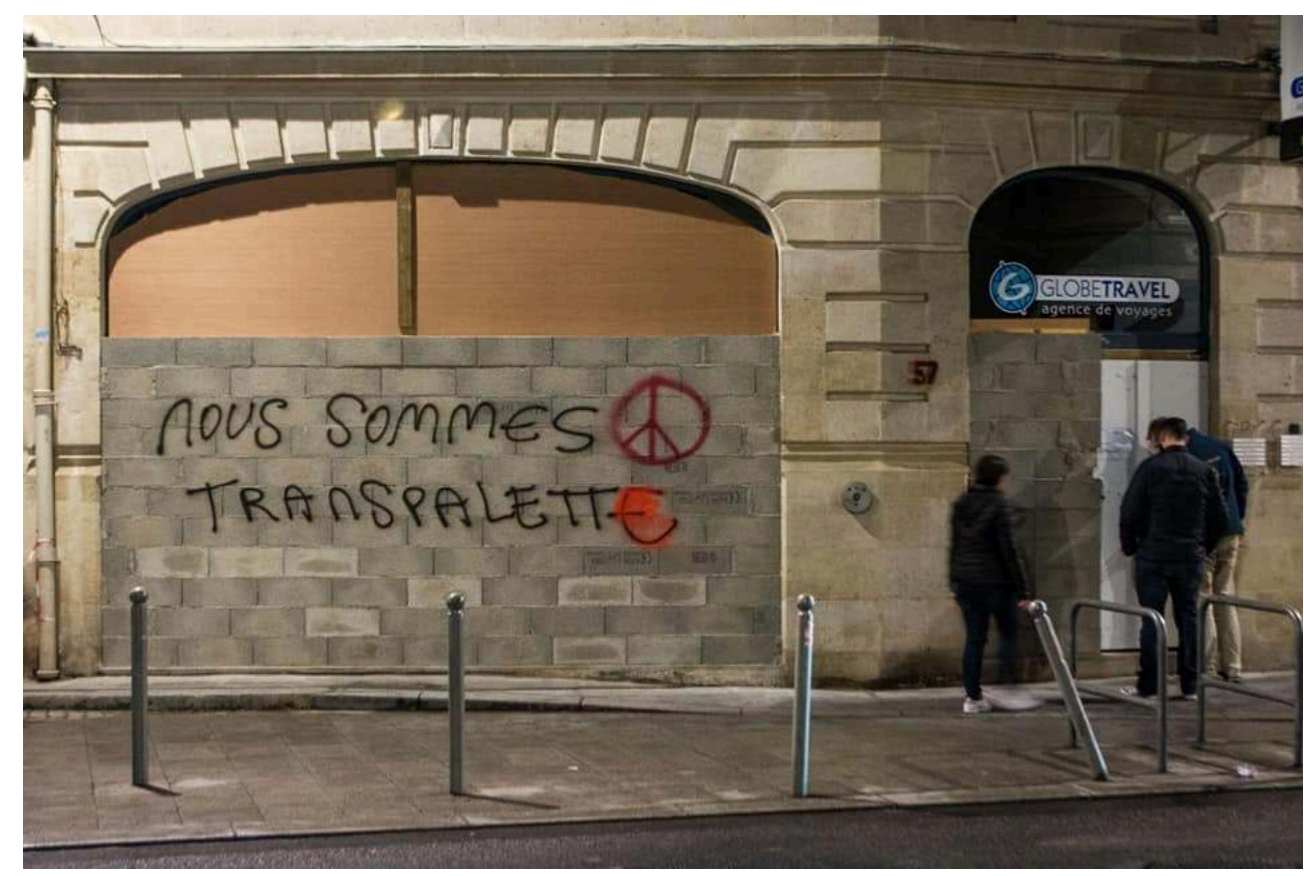

Source : Tumblr, La rue ou rien, https://larueourien.tumblr.com/.

Le présent parcours est, bien entendu, fragmentaire: il ne cherche ni à épuiser la totalité des textes d'Odezenne ni à traiter l'ensemble des thématiques éprouvées par le groupe, et il présente l'inconvénient de porter un regard herméneutique sur une œuvre qui n'est pas achevée. Gageons toutefois que les tendances ici observées en trois temps - depuis l'émergence favorisée par une rhétorique métaplasmique et potache fonctionnant comme droit d'entrée dans le champ jusqu'au développement d'une poétique de l'engagement sociopolitique, d'abord globale avant de se fixer sur des causes et cibles spécifiques, en passant par l'affirmation d'une axiologie partagée entre hédonisme et importance des liens forts - permettent de saisir les fondements d'une poétique développée par un groupe pour se donner les moyens d'exister, de se positionner et de se singulariser dans le milieu du rap, sans se mêler des réseaux et de la mythologie inhérents à ce dernier pour se ménager la marge de manœuvre la plus large possible.

\section{BIBLIOGRAPHIE}

Barret, Julien, 2008, Le Rap ou l'artisanat de la rime, Paris, L'Harmattan.

Bonhomme, Marc, 2005, Pragmatique des figures du discours, Paris, Champion. 
Groupe $\mu, 1970$, Rhétorique générale, Paris, Seuil.

Hammou, Karim, 2009, « Des raps en français au "rap français”. Une analyse structurale de l'émergence d'un monde social professionnel », Histoire \& mesure, vol. XXIV, n 1, p. 73-108, [En ligne], https://doi.org/10.4000/histoiremesure.3889.

Hammou, Karim, 2012, Une histoire du rap en France, Paris, La Découverte.

Pecqueux, Anthony, 2007, Voix du rap. Essai de sociologie de l'action musicale, Paris, L'Harmattan.

Pecqueux, Anthony, 2008, «Articuler nonchalamment la langue française dans la chanson, XIX ${ }^{\mathrm{e}}$ $\mathrm{xxi}^{\mathrm{e}}$ siècles : des diseuses au slam », Université d'automne de la chanson, Marseille, France, [En ligne], http://www.lehall.com/galerie/universitemarseille/?p=685, consulté le 12 juillet 2021.

Roquebert, Corentin, 2020, « Le capital social des rappeurs : les featurings entre gains de légitimités et démarche d'authentification professionnelle », Volume !, vol. 17, nº 2, p. 61-81, [En ligne], https://doi.org/10.4000/volume.8537.

Saint-Amand, Denis, 2016, « "Morts avec supplément frites”. Invectif et logique conflictuelle dans le champ du rap français ", Études de Lettres, $\mathrm{n}^{\circ}$ 3, Les Voies contemporaines de l'oralité, p. 57-71, [En ligne], https://doi.org/10.4000/edl.1206.

Saint-Amand, Denis, 2019, « Parce que c'est notre rejet. Poétique des Gilets jaunes », AOC, $1^{\mathrm{er}}$ février 2019, [En ligne], https://aoc.media/analyse/2019/07/30/parce-que-cest-notre-rejetpoetique-des-gilets-jaunes/, consulté le 12 juillet 2021.

Saint-Amand, Denis, 2019, Le Style potache, Genève, La Baconnière.

Saint-Amand, Denis, 2021, « 1871 raisons d'y croire. Logiques et imaginaire des Gilets jaunes », Nineteenth-Century French Studies, vol. 49, n 3-4, p. 374-395, [En ligne], https://doi.org/10.1353/ ncf.2021.0015.

\section{NOTES}

1. Voir aussi Saint-Amand (2016).

2. La popularité de Bigflo \& Oli les a conduits à réaliser le générique du film La Cartouche du YouTubeur Cyprien Iov (2016), à apparaître dans la comédie Alad'2 de Lionel Steketee (2018) ou à siéger dans le jury du télé-crochet The Voice Belgique.

3. Pour les Casseurs Flowters, voir des titres comme "Regarde comme il fait beau ", "Deux connards dans un abribus", «Greenje et Orselane ", "À l'heure où je me couche », « Quand ton père t'engueule », «J'essaie, j'essaie », « Si facile », « Inachevés ».

4. Dès la fin des années 1990, Caillet et Lucchini fondent le groupe Satanic Spirit, qu'ils abandonnent quand le premier part étudier à l'étranger. En 2003, leurs retrouvailles sont l'occasion d'un changement de style et de nom: ils se rebaptisent O2zen, détournant le nom de leur ancienne proviseure, et, peu après, sont rejoints par Cormary.

5. Invités à se prononcer sur l'inévitable question des influences, les membres du groupe évoquent notamment Rage Against the Machine, Radiohead, Pearl Jam, Nirvana, KoRn, Grandaddy, The Smashing Pumpkins, les Red Hot Chili Peppers, Jimi Hendrix, The Doors, David Bowie, Bob Dylan et Pink Floyd. Voir Azzedine Fall, « En écoute : tous les groupes qui ont influencé Odezenne », Les Inrocks, [En Ligne], 4 novembre 2015, 
https://www.lesinrocks.com/2015/11/04/musique/musique/en-ecoute-les-groupesqui-ont-influence-odezenne/, consulté le 12 juillet 2021.

6. Voir Hammou (2009) et Roquebert (2020).

7. Cette dimension devrait faire l'objet d'une approche plus spécifique, étudiant en détail les différents rouages de cette chaîne de production et de diffusion.

8. «Le métaplasme est une opération qui altère la continuité phonique ou graphique du message, c'est-à-dire la forme de l'expression en tant qu'elle est manifestation phonique ou graphique» (Groupe $\mu 1970$ : 50). À la traditionnelle appréhension de la figure de style comme " écart ", nous associons ici sa saisie comme « saillance ", suivant les propositions de Marc Bonhomme (2005). Julien Barret (2008) fait des métaplasmes et, en particulier, de la paronomase - l'un des rouages majeurs du rap français.

9. Je me permets de renvoyer à mon essai Le Style potache (2019).

10. Le film Le Joli Mai (1963) donne à voir le Paris en transformation de l'après-guerre d'Algérie, dans lequel se croisent des habitants partagés entre la reconquête de leur quotidien et la honte du conflit passé.

11. Le mouvement des Gilets jaunes a pris forme à l'automne 2018 à partir d'une occasion révélatrice d'un malaise (l'annonce de l'augmentation de la taxe intérieure de consommation sur les produits énergétiques ou TICPE), avant de développer son cahier de doléances en dépassant ce seul grief pour mieux l'articuler à d'autres signes d'une crise sociale et politique mis en lumière au fil des échanges et réflexions des insurgés (exigence de rétablissement de l'impôt sur la fortune (ISF), maintien des services publics, hausse du pouvoir d'achat, volonté d'amélioration de la démocratie représentative, etc.). Le nom et l'emblème choisis par les membres du mouvement, le gilet de haute visibilité de couleur jaune, trouvaient leur origine dans l'opposition fondatrice au TICPE; elle se fonde sur un rapport métonymique avec le véhicule personnel visé par la taxe annoncée et mise en outre sur la fonction première du gilet, qui consiste à protéger celui qui le porte en améliorant sa visibilité.

12. Sébastien Crépel, "Les sales méthodes de la BNP pour réduire ses effectifs", L'Humanité, jeudi 2 mai 2019, [En ligne], https://www.humanite.fr/article-sanstitre-671675, consulté le 12 juillet 2021.

13. Maxime Vaudano, Jérémie Baruch et Anne Michel, "Panama papers": les pratiques offshore opaques du Crédit agricole et de la BNP », Le Monde, 11 mai 2016.

14. "L'argent", dans ce cas, renvoie aux sommes évoquées lors des révélations du Canard enchaîné au sujet de François Fillon, alors candidat à l'élection présidentielle, en janvier 2017: il était question des salaires d'assistants parlementaires reçus par sa femme Penelope, sa fille Marie et son fils Charles, des costumes offerts par Robert Bourgi et des bénéfices de sa société de consulting. Voir Isabelle Barré, Hervé Liffran et Christophe Nobili, «Pour Fillon, Penelope est un bon filon », Le Canard enchaîné, $\mathrm{n}^{\circ}$ 5022, 25 janvier 2017 ; Isabelle Barré, Hervé Liffran et Christophe Nobili, «Qui veut gagner

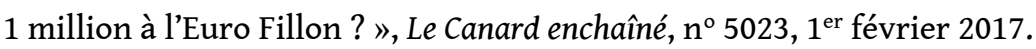

15. Laurent Hoebrechts, «"Bleu fuchsia" c'est notre côté gilets jaunes!", Focus Vif, 26 septembre 2019, [En ligne], https://focus.levif.be/culture/musique/odezenneattitude-bleu-fuschia-c-est-notre-cote-gilets-jaunes/article-normal-1194627.html, consulté le 12 juillet 2021.

16. Le Tumblr La Rue ou rien collecte des centaines de productions écrites liées au mouvement, déclinées sous la forme de tags (principalement), affiches, fresques, 
pancartes et autres banderoles. Voir https://larueourien.tumblr.com/. Sur les écrits sauvages des Gilets jaunes, je me permets de renvoyer à mes articles «Parce que c'est notre rejet. Poétique des Gilets jaunes » (2019) et « 1871 raisons d'y croire. Logiques et imaginaire des Gilets jaunes » (2021).

\section{RÉSUMÉS}

Depuis le milieu des années 2000, le groupe français Odezenne se distingue par son investissement de circuits de production et diffusion parallèles, et s'est en outre forgé une identité propre en misant sur une écriture singulière, affermie au fil des productions : aux virtuosités métaplasmiques des premières compositions succède l'affirmation d'une axiologie hédoniste et, surtout, d'une éthique de l'attention avant que, dès l'EP « Rien », ne se développent progressivement les signes d'un engagement social. Ces différentes orientations ont en commun d'être articulées à l'humour, qui occupe une place de prédilection dans la dynamique et l'imaginaire du groupe.

Since the mid-2000s, Odezenne has explored alternatives labels and broadcasts; it has also developed its very own identity through an original way of writing, that has strengthened over time. As the first compositions were rhetorical displays (with a particular taste for metaplasm), they were followed by tracks aiming to illustrate an axiology based on hedonism and, above all, an ethics of care; since the EP "Rien" (2014), the band also explores socio-political commitment. All these options have in common that they let the band diversify its taste for humor, which is central in its poetics.

\section{INDEX}

Keywords : Odezenne, poetics, rap, rhetoric, engagement, humor

Mots-clés : Odezenne, poétique, rap, rhétorique, engagement, humour

\section{AUTEUR}

DENIS SAINT-AMAND

FNRS - UNamur (NaLTT) 